\title{
Abordagem na educação sexual de adolescentes em ambiente escolar: relato de experiência
}

Approach in sexual education of adolescents in school environment: experience report

Luciana Santos Ferreira $^{1}$; Maria Graziélle Bossi da Silva ${ }^{2}$

${ }^{1}$ Pós-graduanda em Obstetrícia pela IProfit; Jequié, Bahia, Brasil, 45200-000, lulucyferreira@hotmail.com

Maria Graziélle Bossi da Silva

${ }^{2}$ Mestre em Ensino, Filosofia e História das Ciências-UFBA/UEFS

Jequié, Bahia, Brasil, 45200-000, grazibossi@hotmail.com

\section{Resumo}

A educação em saúde é a principal ferramenta no desenvolvimento de ações para a prevenção de Infecções Sexualmente Transmissíveis (IST). A adolescência é caracterizada por vulnerabilidade, por ser uma fase da vida onde as subversões são no âmbito social, psicológico, físico e emocional. Este trabalho tem o objetivo de relatar a experiência de acadêmicas do curso de Enfermagem da Faculdades Unidas de Pesquisa, Ciências e Saúde (FAPEC) em atividade educativa sobre IST/HIV-AIDS. A atividade ocorreu em formato de educação continuada, desenvolvida no estágio supervisionado I, em uma escola vinculada a ESF. O público alvo constituiu-se por alunos do $9^{\circ}$ ano do Ensino Fundamental. Utilizou-se estratégia de dinâmica em grupo para abordar o tema "complicações e formas de prevenção das IST". Aplicou-se questionário pré-teste e constatou-se desinformação frente à questionamentos básicos acerca das IST e uso do preservativo. A atividade proposta simulou a cadeia de transmissão das IST/HIV-AIDS, levando-os a refletir sobre a vivência sexual responsável, como fator condicionante de uma vida saudável. A experiência de ensino mostrou que é de suma importância a presença de profissionais de saúde em ambiente escolar para contribuir com a disseminação práticas saudáveis no autocuidado e a importância na prevenção de agravos.

Palavras chave: Adolescentes; Educação em Saúde; Infecção Sexualmente Transmissível; Enfermagem. 


\begin{abstract}
Health education is the main tool in the development of actions for the prevention of Sexually Transmitted Infections (STIs). Adolescence is characterized by vulnerability, as it is a phase of life where subversions are social, psychological, physical and emotional. This paper aims to report the experience of nursing students from the Faculdades Unidas de Pesquisa, Ciências e Saúde (FAPEC) in educational activity on STI / HIV-AIDS. The activity took place in a continuing education format, developed in supervised internship I, in a school linked to the ESF. The target audience consisted of students from the 9th grade of elementary school. A group dynamics strategy was used to address the theme "complications and ways of preventing STIs". A pre-test questionnaire was applied and there was disinformation regarding basic questions about STIs and condom use. The proposed activity simulated the chain of transmission of STIs / HIV-AIDS, leading them to reflect on responsible sexual experience, as a conditioning factor for a healthy life. The teaching experience has shown that the presence of health professionals in the school environment is of utmost importance to contribute to the dissemination of healthy practices in self-care and the importance in preventing diseases.
\end{abstract}

Keywords: Adolescents; Health education; Sexually Transmitted Infection; Nursing

\title{
1. Introdução
}

A lei $n^{\circ} 8.069$ de 13 de julho de 1990, que rege o Estatuto da Criança e do Adolescente (ECA), versa que a adolescência é a etapa da vida compreendida entre a infância e a fase adulta, de 12 a 18 anos de idade, marcada por um complexo processo de crescimento e desenvolvimento biopsicossocial (BRASIL, 2019). Essa etapa é marcada por inúmeras alterações nos aspectos físico, emocional, psicológico e, por isso, merece atenção diferenciada para que sejam detectados precocemente comportamentos considerados vulneráveis, no intuito de orientá-los quanto a importância de sua responsabilização na prevenção de agravos.

Um dos principais problemas de saúde pública referente aos adolescentes na atualidade é o crescente aumento dos riscos de contrair 'Infecções Sexualmente Transmissíveis' (IST), uma vez que, a atividade sexual está sendo iniciada cada vez mais precocemente, e sem a devida orientação. Seja por medo, vergonha, imaturidade, descrença na existência das ISTs ou desconhecimento das formas de prevenção, os adolescentes assumem comportamentos para os quais ainda não estão totalmente preparados (MONTEIRO, 2019). 
De acordo com Krabbe e colaboradores (2016), na maioria das vezes, a primeira relação sexual não é um evento planejado, ocorrendo em algum momento inesperado e sem preparação. A falta de conhecimento sobre a vulnerabilidade deixa o jovem exposto a riscos. Desta forma, se faz necessário adotar medidas de conscientização e elaboração de planos de educação em saúde para se abordar com os adolescentes e esclarecer sobre os riscos associados à vida sexual precoce.

Por não estarem informados ou subsidiados por informações consideradas corretas, os adolescentes aprendem e disseminam informações inadequadas e preconceitos que, se somados ao comportamento onipotente característico dessa fase, contribuem para que as experiências sexuais possam condicionar a riscos (COSTA et al, 2001). Diante desses comportamentos, os pais, professores e profissionais de saúde devem atuar na educação sexual conjuntamente, no intuito de orientar uma vivência afetiva e sexual responsável, para minimizar ou evitar os riscos.

Para a efetivação de uma educação em saúde que contribua no desenvolvimento de práticas críticas e reflexivas, é necessária a definição de campos de ação para a promoção da saúde como, por exemplo, a construção de políticas públicas saudáveis; reorientação dos serviços de saúde; desenvolvimento de habilidades individuais; e reforço da ação comunitária, por meio da responsabilidade social. Essa opção deve estar fundamentada na análise da realidade que se faz mediante a identificação de problemas e das necessidades de saúde da população, para então estimular a reflexão crítica da realidade, sendo indispensáveis ações de educação em saúde voltadas para a melhoria dos determinantes da saúde (ALVES \& AERTS, 2011).

O incentivo para a elaboração deste estudo emergiu após a aplicação de uma atividade educativa em saúde, cujo tema foi escolhido em reunião entre parte da equipe de acadêmicas, o articulador do estágio, e a coordenadora da ESF, como uma estratégia de abordar a temática com alunos de assuntos condizentes com essa fase da vida que, é repleta de mudanças e, requer atenção especial por parte dos profissionais envolvidos. Uma vez que as escolas não dispõem em seu quadro de professores profissionais com disponibilidade para atuar de forma preventiva na questão da sexualidade esse trabalho tornou-se de grande 
relevância, o que pôde ser constatado mediante a receptividade da coordenadora escolar.

Diante do exposto, o objetivo deste trabalho é relatar a experiência vivida na aplicação de atividade educativa sobre infecções sexualmente transmissíveis para adolescentes de 12 à 16 anos, estudantes do $9^{\circ}$ ano, avaliando seu nível de conhecimento prévio acerca do tema abordado e oportunizar o desenvolvimento de pensamento crítico a fim de conscientizá-los quanto sua responsabilidade no autocuidado.

\section{Material e Métodos}

Trata-se de um relato de experiência de uma atividade educativa sobre IST, que foi desenvolvida por acadêmicas de Enfermagem do IX semestre da Faculdades Unidas de Pesquisa, Ciências e Saúde (FAPEC) no município de Jequié-BA.

A atividade foi realizada com estudantes do $9^{\circ}$ Ano do Ensino Fundamental, de uma escola municipal situada na área de abrangência da ESF vinculada à FAPEC, onde as acadêmicas cumpriram a carga horária do Estágio Supervisionado I. A atividade ocorreu no período matutino do dia 28 de março de 2018, com a efetiva participação de 31 alunos, na faixa etária entre 12 e 16 anos, dos quais vinte e três eram do sexo feminino e oito do sexo masculino.

Tomando como base as metodologias ativas, as atividades realizadas com os alunos foram adaptadas de Costa (2014), com o intuito de estimular os adolescentes à capacidade de desenvolver um pensamento crítico. Inicialmente foi aplicado um questionário pré-teste, com seis questões de múltipla escolha, em que havia apenas uma resposta correta para cada questão. A finalidade do pré-teste foi identificar o nível de conhecimento dos alunos no que se refere à saúde sexual e reprodutiva.

Na sequência, foi realizada uma dinâmica de grupo com o objetivo de facilitar o reconhecimento de comportamentos vulneráveis por parte dos adolescentes, e a identificação da cadeia de transmissão das IST. Além disso, a dinâmica tinha como intuito levá-los a refletir sobre a importância da vivência sexual responsável e alertar sobre os perigos da multiplicidade de parceiros e do sexo desprotegido.

Para a realização da dinâmica, foi entregue a cada aluno um papel com uma figura 
geométrica (triângulo, quadrado ou círculo) e cinco espaços em branco. Foi orientado para que eles dançassem ao som de uma música e a cada pausa copiassem a figura geométrica do colega que estava parado ao lado, nos referidos espaços. Houve pausa por cinco vezes, e a cada pausa era copiada uma figura em locais diferentes do papel (lado direito, lado esquerdo, e em frente). Ao término da dinâmica foi exposta a seguinte legenda no quadro: o triângulo equivalia a uma pessoa portadora de HIV, o círculo representava uma pessoa sadia, e o quadrado uma pessoa com alguma IST.

Por não se tratar de pesquisa envolvendo diretamente seres humanos não houve necessidade de submeter esse estudo ao Comitê de Ética em Pesquisa.

\section{Resultados}

Após a aplicação da dinâmica em grupo, todos os alunos representavam pessoas contaminadas com HIV, IST ou HIV e IST (fig 1), ou seja, não restaram pessoas saudáveis. Os três alunos que estavam com a figura do triângulo (HIV) foram capazes de "contaminar" 25 pessoas. Os sete que estavam com o quadrado (IST) “contaminaram” 28 pessoas. Esse tipo de dinâmica demonstra quão perigoso pode ser a multiplicidade de parceiros com sexo desprotegido.

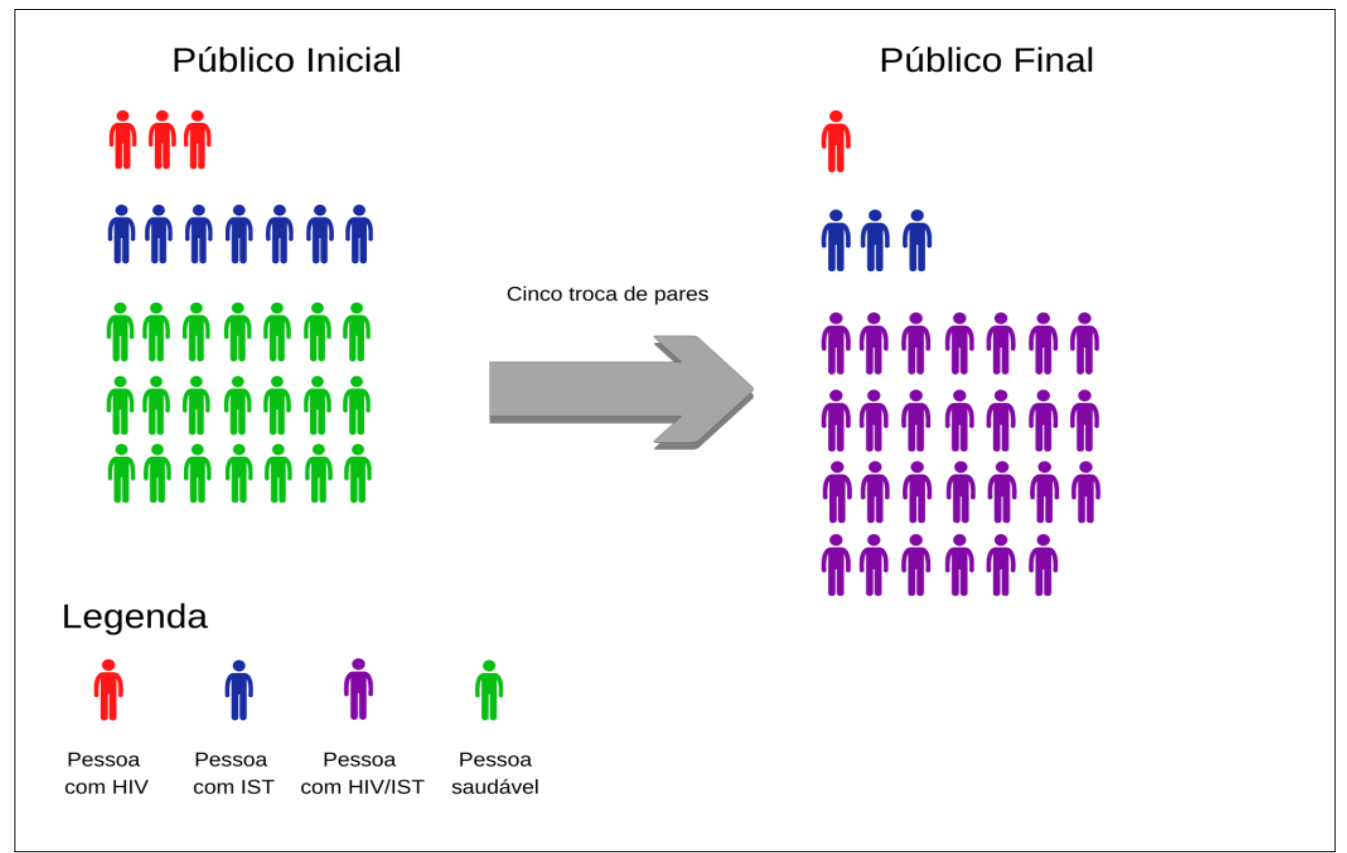

Figura 1. Representação da distribuição da condição das pessoas antes e após a realização da dinâmica em grupo. 
Além de exemplificar o quanto é alta a probabilidade de contaminação quando se pratica sexo desprotegido, a atividade educativa também serviu para mostrá-los como funciona a cadeia de transmissão das IST, os perigos na rotatividade de parceiros e a importância de práticas seguras nas relações sexuais. Ao longo da discussão foi enfatizado que as pausas na dança representam trocas de parceiros ou namorados durante a vida do ser humano. Do mesmo modo que os desenhos foram copiados, de maneira descontraída, existe a possibilidade de contrair uma IST ao manter contato com outra pessoa sem usar a prevenção.

Por se tratar de uma fase delicada na vida do ser humano, a adolescência merece um olhar diferenciado por parte dos profissionais de saúde. Se por um lado eles têm a informação ao alcance das mãos, através dos meios de comunicação, por outro lado o conflito interno vivenciado por eles acaba por não permitir que se informem com quem realmente possa lhes dar orientações, como os pais, profissionais da educação e da saúde. Isso acaba por confundir sua mente e muitas vezes o leva a um caminho tortuoso, onde consequências são danosas à sua saúde. De acordo com Girondi et al. (2006), esta fase é marcada por duas aquisições importantes: a capacidade reprodutora e a identidade pessoal.

A Estratégia de Saúde da Família (ESF), desde sua implantação vem sendo reformulada e aprimorada para que os serviços oferecidos à população estejam condizentes com a realidade da comunidade. Nesse contexto, a Educação em saúde é uma aliada na prevenção de agravos, sobretudo quando se estabelece parceria entre ESF e educação. Encontros estabelecidos entre profissionais da saúde em ambiente escolar proporcionam uma troca de experiências coletivas que levam o adolescente a refletir sobre suas práticas. Santos, Bertolozzi e Hino (2010), afirmam que, profissionais de saúde nem sempre estão preparados para lidar com as necessidades de saúde dos usuários.

De acordo com Miltre et al. (2006), o grande desafio deste século está na perspectiva de se desenvolver a autonomia individual em íntima coalizão com o coletivo. Se por um lado a educação deve levar o indivíduo a ampliar seus conhecimentos, por outro deve permitir à conscientização da importância no autocuidado, fazendo com que ele entenda que é um ser crítico, que a responsabilidade deve ser direta na prevenção de agravos, proteção individual e coletiva. Portanto, um dos seus méritos está na tendência à busca de métodos inovadores, 
que admitam uma prática educativa moral, crítica, reflexiva e transformadora, capaz de permitir a transformação do homem inscrito na lógica da ação-reflexão-ação.

Após a aplicação da dinâmica e análise das reflexões, foi realizada uma apresentação de slides, com ênfase em demonstrar informações, imagens de órgãos sexuais afetados pelas IST mais comuns. Nesse momento, os alunos puderam esclarecer suas dúvidas e demonstraram surpresa ao ver as imagens, alguns inclusive demonstraram sentimentos de repulsa e asco. As principais e mais frequentes dúvidas estavam relacionadas ao fato direto de transmissão das IST, vias e opção sexual. Foi questionado, por exemplo, se uma profissional do sexo tem chance aumentada de contrair IST, se o sexo oral pode transmitir alguma IST, dentre outras.

Ao final da apresentação foi aplicado o questionário pós-teste a fim de avaliar se houve mudança no conhecimento dos estudantes em relação aos temas abordados. Nesse questionário foram aplicadas as mesmas questões utilizadas no pré-teste. A análise das respostas do pré-teste demonstra que mesmo as perguntas sendo consideradas fáceis, os adolescentes apresentavam conhecimentos de senso comum. No pós-teste houve uma pequena evolução em algumas respostas, enquanto outras se mantiveram incorretas.

Diante disso, constatou-se que apenas um encontro não é suficiente para que ocorra a aprendizagem significativa dos conteúdos explanados, apontando para a necessidade de um trabalho mais aprofundado para que os estudantes consigam internalizar os conteúdos abordados e tomar decisões mais conscientes no que diz respeito a sua sexualidade.

Ferreira et al. (2014), afirmam que o objetivo da educação é oportunizar momentos de reflexões e ações capazes de possibilitar às pessoas um aprendizado consciente, sem a intenção de controlar suas vidas. Sendo assim, a educação em saúde pode ser considerada como uma oportunidade de ampliação no conhecimento de práticas educativas, que visem não só o autocuidado, como também aprendizados conscientes e preventivos. O diálogo face a face permite troca de experiências, esclarecimentos de dúvidas e desenvolvimento do pensamento crítico reflexivo.

Através deste trabalho foi possível oportunizar aos adolescentes uma troca de conhecimento de maneira descontraída e leve; aproveitando seu conhecimento prévio na 
explanação do assunto; demonstrar através de imagens reais as consequências de contrair IST, expondo-lhes fotos com evolução das IST; e estimular o desenvolvimento de um senso crítico reforçando a eles a importância de aquisição de senso de responsabilidade no autocuidado e prevenção.

Há, portanto uma importância primordial na flexibilização ao se planejar atividades voltadas para a educação em saúde, sobretudo na educação sexual dos adolescentes, devendo-se sempre que possível inserir os que estão vinculados aos grupos escolares no planejamento das ESF. Uma vez que as escolas fazem parte da sua área de abrangência, tais atividades podem aumentar o conhecimento acerca da importância de uma vida sexual protegida, segura e devidamente acompanhada para diminuir as chances no aumento das IST, de gestações indesejadas entre outras questões relacionadas à saúde.

Atrair e manter a atenção de adolescentes em ambiente escolar não é uma tarefa fácil, sobretudo quando se trata de profissionais que não fazem parte da sua rotina diária. Mesmo assim, encontros como esses se fazem necessários para que seja proporcionado conhecimento no autocuidado cada vez mais cedo entre os jovens, uma vez que os mesmos estão iniciando sua vida sexual precocemente e de maneira desprotegida e sem orientações adequadas.

Conforme pontua Alvin (2007), o exercício da prática de educação popular em saúde pressupõe abertura, disponibilidade para ouvir o outro, horizontalidade na relação interpessoal e na ação educativa em si, pois, o ato participativo é humanizante. Pode-se concordar que a relação educativa proporciona uma aquisição mútua de conhecimentos, onde educando e educador complementam seus saberes a partir do saber do outro, sempre sendo reformulados os saberes.

\section{Discussão}

A vivência desta atividade mostra que existe a necessidade da presença de profissionais da saúde no ambiente escolar, como uma estratégia que facilite o entendimento da importância de práticas conscientes e seguras nas atividades relacionadas à saúde dos adolescentes, sobretudo no âmbito da educação sexual e reprodutiva.

$\mathrm{Na}$ atualidade, pode-se observar que, devido o advento da tecnologia, a velocidade e 
praticidade com que as informações a cerca da sexualidade chegam ao alcance do público jovem, tem aumentado os riscos de contrair IST/HIV. Faz-se necessário que as ESF estejam presentes nas escolas desenvolvendo atividades voltadas para práticas educativas em saúde sexual, com o objetivo de minimizar estes riscos.

A união entre ESF e Instituições de Ensino Superior é uma parceria eficaz onde ambas tendem a ganhar. A educação em saúde intervém na ampliação do pensamento crítico reflexivo nos adolescentes, facilitando o entendimento da sua responsabilidade no autocuidado, alcançando assim comportamentos preventivos e minimizando riscos de infecções por IST/HIV. Nesse sentido, os acadêmicos tanto podem ajudar, como têm nesse momento oportunidades de vivenciar novas e transformadoras experiências.

\section{Agradecimentos}

A Faculdade Unidas de Pesquisa, Ciências e Saúde e a Equipe de Saúde da Família (ESF) Senhorinha Ferreira de Araújo, assim como a equipe da Escola de Curral Novo, pelo suporte na execução desta pesquisa. Ao enfermeiro Marcus Rabello que oportunizou a execução desta pesquisa.

\section{Referencias}

Alves, G. G., \& Aerts, D. (2011). As práticas educativas em saúde e a Estratégia Saúde da Família. Ciência \& Saúde Coletiva, v. 16, p. 319-325. https://doi.org/10.1590/S1413$\underline{81232011000100034 .}$

Alvin, N. A. T., \& Ferreira, M. A. (2007) Perspectiva problematizadora da educação popular em saúde e a enfermagem. Texto Contexto Enfermagem, v. 16, p. 315-319. https://doi.org/10.1590/S0104-07072007000200015.

Brasil. Ministério da Saúde. (2019). Estatuto da Criança e do Adolescente - Lei 8069/90 Lei no 8.069, de 13 de julho de 1990, atualizada em 16 de março de 2019. Disponível em: https://doi.org//www.gov.br/mdh/pt-br/centrais-de-conteudo/crianca-e-

adolescente/estatuto-da-crianca-e-do-adolescente-versao-2019.pdf. Acessado em $28 / 10 / 2020$

Costa, M. C. O; Lopes, C. P. A; Souza, R. P. et al. (2001). Sexualidade na adolescência: desenvolvimento, vivência e propostas de intervenção. Jornal da pediatria, 217-224.2001. Disponível em: https://doi.org//www.jpde.com.br/conteudo/01-77-s217/port.pdf 
Costa, D. F. (2014). Os desafios da Escola Pública Paranaense na perspectiva do professor PDE. $2014 . \quad$ Disponível em: https://doi.org//www.diaadiaeducacao.pr.gov.br/portals/cadernospde/pdebusca/producoes pde/2014/2014_uel_bio_pdp_daniele_fernanda_costa.pdf. Acessado em 28/10/2020

Ferreira, V. F., Rocha, G. O., Lopes, et al. (2014). Educação em Saúde e Cidadania: Revisão Integrativa. Trabalho Educação em Saúde, 363-378. 2014. https://doi.org/10.1590/S198177462014000200009.

Girondi, J. B. R; Nothaft, S. C. S; Mallmann, F. M. B. (2006). A metodologia problematizadora utilizada pelo enfermeiro na educação sexual de adolescentes. Cogitare Enfemagem, v. 11, p. 161-165. http://dx.doi.org/10.5380/ce.v

Krabbe, E. C; et. al; (2016). Escola, Sexualidade, práticas sexuais e vulnerabilidades para as infecções sexualmente transmissíveis (IST). Revista Interdisciplinar de Ensino, Pesquisa e extensão, $\mathrm{v}, 4, \mathrm{p} .1-84$.

Mitre, S. M; et. al. (2006). Metodologias ativas de ensino-aprendizagem na formação profissional em saúde: debates atuais. Ciência e Saúde Coletiva. DOI: 10.5205/reuol.476742136-1-ED.0712esp201311

Monteiro, C. J; \& Jesus, T. B. Avaliação do nível de conhecimento dos jovens a respeito das manifestações orais de infecções sexualmente transmissíveis. Departamento de Odontologia de Lagarto, Universidade Federal de Sergipe. 43p. 2019.

Santos, P. T; Bertolozzi, M. R; Hino, P. (2010). Necessidades de saúde na atenção primária: percepção de profissionais que atuam na educação permanente. Acta Paulista de Enfermagem, v. 23, p. 788-795. https://doi.org/10.1590/S0103-21002010000600012 